\title{
Auditing of Storage Security on Encryption
}

\author{
V. Sujatha1)
}

\begin{abstract}
Cloud storage services became progressively fashionable. attributable to the importance of privacy, several cloud storage secret writing schemes are projected to guard information from those that don't have access. All such schemes assumed that cloud storage suppliers area unit safe and can't be hacked; but, in follow, some authorities (i.e., coercers) might force cloud storage suppliers to reveal user secrets or confidential information on the cloud, therefore altogether circumventing storage secret writing schemes. during this paper, we have a tendency to gift our style for a brand new cloud storage secret writing theme that allows cloud storage suppliers to make convincing faux user secrets to guard user privacy. Since coercers cannot tell if obtained secrets area unit true or not, the cloud storage suppliers make sure that user privacy continues to be firmly protected. Most of the projected schemes assume cloud storage service suppliers or reliable third parties handling key management square measure reliable and cannot be hacked; however, in follow, some entities would possibly intercept communications between users and cloud storage suppliers then compel storage suppliers to unhitch user secrets by exploitation government power or different suggests that. throughout this case, encrypted info square measure assumed to be known and storage suppliers square measure requested to unhitch user secrets.
\end{abstract}

Keywords: Fashionable,Tending, Unharness, Encrypted, Compel.

\section{Existing System}

Most previous refutable secret writing schemes, we have a tendency to don't use semitransparent sets or simulatable public key systems to implement deniability. Instead, we have a tendency to adopt the concept projected in with some enhancements. we have a tendency to construct our refutable secret writing theme through a dimensional house. All information area unit encrypted into the dimensional house. solely with the right composition of dimensions is that the original information available. With false composition, ciphertexts are going to be decrypted to preset faux information. the knowledge process the scale is unbroken secret. we have a tendency to create use of Composite order additive teams to construct the dimensional house. we have a tendency to conjointly use chameleon hash functions to create each true and faux messages convincing.

Received(January 26, 2017), Review Result(1st: February 13, 2017, 2nd: March 20, 2017), Accepted(April 10, 2017)

${ }^{1}$ Department of Computer Science and Engineering Vignan's Nirula Institute of Technology \& Science for Women, Pedapalakaluru,Guntur.

email: sujiekkurthi@gmail.com 


\section{Proposed System}

Techniques utilized in previous refutable secret writing schemes, we have a tendency to build 2 secret writing environments at identical time, very similar to the concept projected in We build our theme with multiple dimensions whereas claiming there's just one dimension. This approach removes obvious redundant elements in . we have a tendency to apply this idea to $\mathrm{AN}$ existing $\mathrm{ABE}$ theme by commutation prime order teams with Composite order teams. Since the bottom ABE theme will write one block when, our refutable [1]CPABE is definitely a blockwise refutable secret writing theme. tho' the additive operation for the Composite order cluster is slower than the prime order cluster, there area unit some techniques which will convert AN secret writing theme from Composite order teams to prime order teams for higher machine performance.

\section{Advantages:}

\section{(3) Blockwise refutable ABE:}

This reduces the continuance range from the block size to the key size. tho' bitwise refutable secret writing is a lot of versatile than blockwise refutable secret writing in "cooking" faux information, once considering cloud storage services, blockwise secret writing is way a lot of economical in use.

\section{(3) Consistent Environment:}

we build a standardized setting for our refutable secret writing theme. By consistent setting, we have a tendency to implies that one secret writing setting may be used for multiple secret writing times while not system updates.

\section{(1) Deterministic Decryption:}

The conception of our refutable theme is totally different than these schemes delineated on top of. Our theme extends a pairing $\mathrm{ABE}$, that contains a settled coding algorithmic program

\section{Drawback Statement}


Most refutable public key schemes area unit bitwise, which implies these schemes will solely method one bit a time; so, bitwise refutable secret writing schemes area unit inefficient for real use, particularly within the cloud storage service case. to unravel this drawback, designed a hybrid secret writing theme that at the same time uses trigonal and uneven secret writing. They use a deniably encrypted plan-ahead trigonal encoding key, whereas real information area unit encrypted by a trigonal key secret writing mechanism. Most refutable secret writing schemes have coding error issues. These errors come back from the designed coding mechanisms. uses the set call mechanism for coding. The receiver determines the decrypted message in keeping with the set call result. If the sender chooses part from the universal set however sadly the element is found within the specific set, then miscalculation happens. identical error happens altogether translucentset- primarily based refutable secret writing schemes.

\section{Scope}

The policy of a file is also denied underneath the request by the client, once terminating the time of the agreement or completely move the files beginning with one cloud then onto subsequent cloud nature's domain. the purpose once any of the on top of criteria exists the policy are going to be unacknowledged and also the key director can completely evacuates the general public key of the associated file. thus nobody will recover the key of a unacknowledged get in future. For this reason we will say the file is definitely erased. To recover the file, the user should evoke the key supervisor to provide the general public key. For that the user should be verified. The key policy attribute primarily based secret writing commonplace is employed for file access that is verified by suggests that of AN attribute connected with the file. With file access management the file downloaded from the cloud are going to be within the arrangement of scan simply or write underpinned. each shopper has connected with approaches for every one file. therefore the right shopper can access the proper file. for creating file access the key policy attribute primarily based secret writing. 


\section{Implementation of modules}

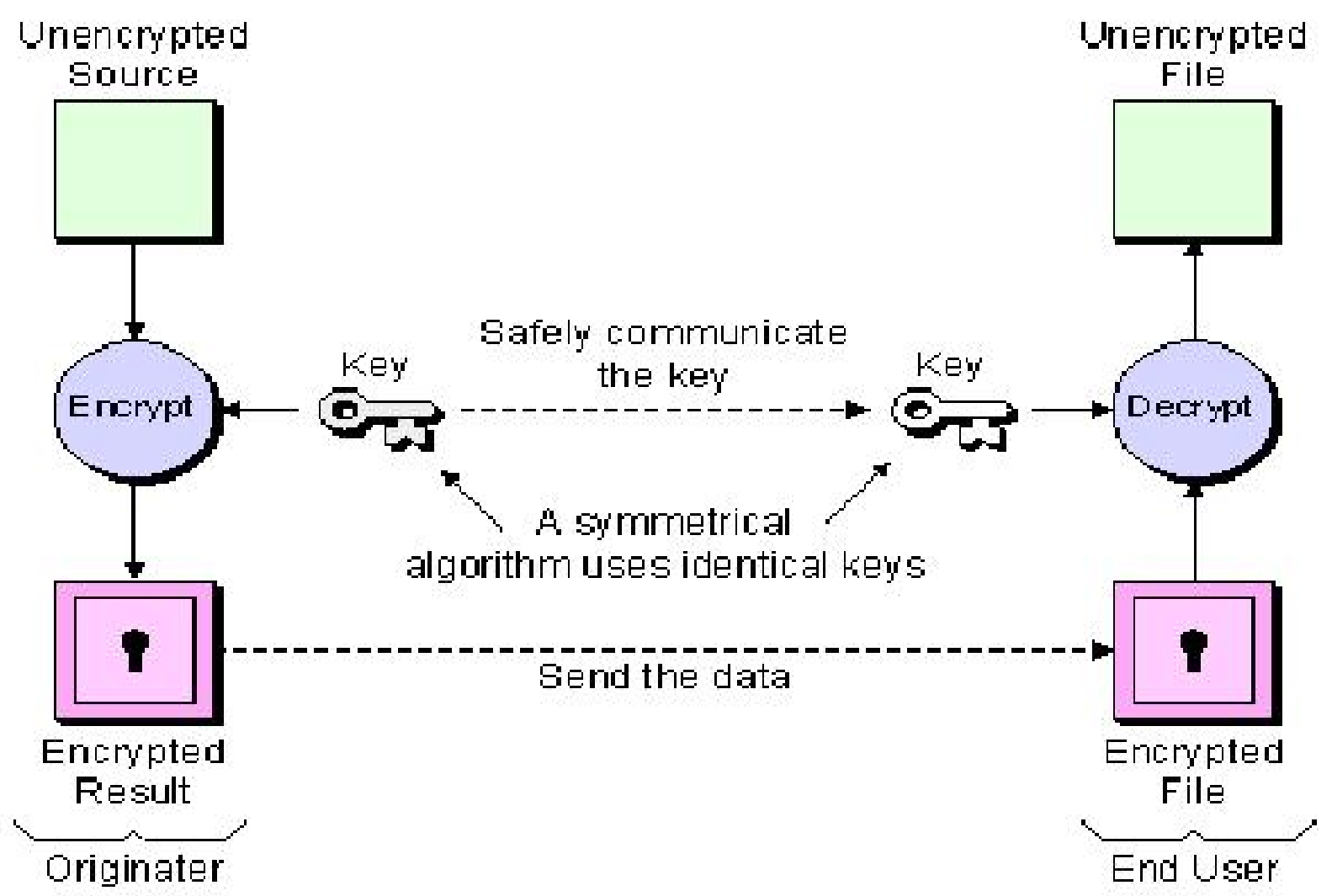

\section{Deniable Encryption:}

Deniable secret writing involves senders and receivers making convincing faux proof of solid information in ciphertexts such outside coercers ar happy. Note that deniability comes from the actual fact that coercers cannot prove the planned proof is wrong and thus haven't any reason to reject the given proof. This approach tries to altogether block coercion efforts since coercers recognize that their efforts are useless. we have a tendency to create use of this concept such cloud storage suppliers will offer audit-free storage services. within the cloud storage situation, information homeowners World Health Organization store their information on the cloud are similar to senders within the confutative secret writing theme. those that will access the encrypted information play the role of receiver within the confutative secret writing theme, together with the cloud storage suppliers themselves, World Health Organization have system wide secrets and should be able to decode all encrypted information. we have a tendency to create use of $\mathrm{ABE}$ characteristics for securing keep information with a fine-grained access 
management mechanism and confutative secret writing to forestall outside auditing.

\section{Composite order linear Group:}

Design a confutative $\mathrm{CP}-\mathrm{ABE}$ theme with Composite order linear teams for building audit-free cloud storage services. Composite order linear teams have 2 enticing properties, specifically sticking and cancelling. we have a tendency to create use of the cancelling property for building a uniform environment; but, freewoman conjointly got wind the vital drawback of process price in relevancy the Composite order linear cluster. The linear map operation of a Composite order linear cluster is far slower than the operation of a major order linear cluster with an equivalent security level. [2] That is, in our theme, a user can pay an excessive amount of time in cryptography once accessing files on the cloud. to form Composite order linear cluster schemes a lot of sensible, into prime order schemes. each sticking and cancelling can't be at the same time achieved in prime order teams in. For an equivalent reason, we have a tendency to use a simulating tool planned to convert our Composite order linear cluster theme to a major order linear cluster theme. This tool relies on twin orthonormal bases and also the mathematical space assumption. totally completely different subgroups ar simulated as different orthonormal bases and thus, [3]by the orthogonal property, the linear operation are off between completely different subgroups. Our formal confutative $\mathrm{CP}-\mathrm{ABE}$ construction technique uses solely the cancelling property of the Composite order cluster.

\section{Attribute-Based Encryption:}

Cloud storage services have quickly become more and more fashionable. Users will store their information on the cloud and access their information anyplace at any time. due to user privacy, the info keep on the cloud is often encrypted and guarded from access by alternative users. Considering the cooperative property of the cloud information, attribute-based secret writing $(\mathrm{ABE})$ is considered one amongst the foremost appropriate secret writing schemes for cloud storage. There ar various $\mathrm{ABE}$ schemes that are planned, including. Most of the planned schemes assume cloud storage service suppliers or trustworthy third parties handling key management ar trustworthy and can't be hacked; but, in follow, some entities might intercept communications between users and cloud storage suppliers and so compel storage suppliers to unharness user secrets by exploitation government power or alternative means that. during this case, encrypted information ar assumed to be glorious and storage suppliers ar requested to 
unharness user secrets. As Associate in Nursing example, in 2010, while not notifying its users, Google discharged user documents to the FBI once receiving a hunt warrant . In 2013, Edward Snowden disclosed the existence of world police investigation programs that collect such cloud information as emails, texts, and voice messages from some technology corporations. Once cloud storage suppliers are compromised, all secret writing schemes lose their effectiveness. although we have a tendency to hope cloud storage suppliers will fight against such entities to take care of user privacy through legal avenues, it's on the face of it a lot of and tougher.

\section{Cloud Storage:}

Cloud storage services became more and more fashionable. due to the importance of privacy, several cloud storage secret writing schemes are planned to safeguard information from those that don't have access. All such schemes assumed that cloud storage suppliers ar safe and can't be hacked; but, in follow, some authorities (i.e., coercers) might force cloud storage suppliers to reveal user secrets or confidential information on the cloud, so altogether circumventing storage secret writing schemes. during this paper, we have a tendency to gift our style for a brand new cloud storage secret writing theme that allows cloud storage suppliers to form convincing faux user secrets to safeguard user privacy. Since coercers cannot tell if obtained secrets are true or not, the cloud storage suppliers make sure that user privacy continues to be firmly protected. Most of the planned schemes assume cloud storage service suppliers or trustworthy third parties handling key management are trustworthy and can't be hacked; but, in follow, some entities might intercept communications between users and cloud storage suppliers and so compel storage suppliers to unharness user secrets by exploitation government power or alternative means that. during this case, encrypted information are assumed to be glorious and storage suppliers are requested to unharness user secrets. we have a tendency to aimed to create Associate in Nursing secret writing theme that might facilitate cloud storage suppliers avoid this plight. In our approach, we provide cloud storage suppliers means that to form faux user secrets. Given such faux user secrets, outside coercers will solely obtained solid information from a user's keep ciphertext. Once coercers assume the received secrets are real, they'll be happy and a lot of significantly cloud storage suppliers won't have unconcealed any real secrets. Therefore, user privacy continues to be protected. this idea comes from a special quite secret writing theme referred to as confutative secret writing. 


\section{Owner Module:}

Owner module is to transfer their files exploitation some access policy. 1st they get the general public key for specific transfer file once obtaining this public key owner request the key key for specific transfer file. exploitation that secret key owner transfer their file.

\section{User Module:}

This module is employed to assist the shopper to look the file victimization the file id and file name .If the file id and name is wrong suggests that we have a tendency to don't get the file, otherwise server raise the general public key and obtain the secret writing file.If $u$ wish the the cryptography file suggests that user have the key key.

\section{Distributed Key Policy Attribute primarily based Encryption:}

$\mathrm{KP}-\mathrm{ABE}$ may be a public key cryptography primitive for one-to-many correspondences. In $\mathrm{KP}-\mathrm{ABE}$, data is related to attributes for every of that a public key half is characterised. The encryptor associates the set of attributes to the message by scrambling it with the examination public key elements. each shopper is appointed Associate in Nursing access structure that is generally characterised as Associate in Nursing access tree over data attributes, i.e., within hubs of the access tree square measure limit doors and leaf hubs square measure connected with attributes. shopper secret key's characterised to mirror the access structure therefore the shopper has the power to decrypt a cipher-text if and simply if the data attributes fulfill his access structure. The projected theme consists of 4 algorithms that is outlined as follows

\section{Setup:}

This formula takes as input security parameters and attribute universe of cardinality N.[4] It then defines a linear cluster of prime quantity. It returns a public key and also the passe-partout that is unbroken secret by the authority party.

\section{Algorithm:}

Deniable (CP-ABE): Our plan-ahead, bideniable, and multi-distributional CP-ABE theme consists of the subsequent algorithms: 
(1) Setup(1) $\rightarrow$ (PP,MSK): This formula takes security parameter as input and returns public parameter PP and system passe-partout MSK.

(1) KeyGen(MSK, S) $\rightarrow$ SK: Given set of attributes S and MSK, this formula outputs personal key SK.

(1) $\operatorname{Enc}(\mathrm{PP}, \mathrm{M}, \mathrm{A}) \rightarrow \mathrm{C}$ : This secret writing formula takes as input public parameter PP, message $M$, and LSSS access structure $A=(M$,$) over the universe of attributes. This formula$ encrypts $\mathrm{M}$ and outputs a ciphertext $\mathrm{C}$, which may be decrypted by people who possess Associate in Nursing attribute set that satisfies access structure A. Note that A is contained in C.

(1) $\operatorname{Dec}(\mathrm{PP}, \mathrm{SK}, \mathrm{C}) \rightarrow$ : This cryptography formula takes as input public parameter PP, personal key SK with its attribute set $S$, and ciphertext $C$ with its access structure $A$. If $S$ satisfies $\mathrm{A}$, then this formula returns $\mathrm{M}$; otherwise, this formula returns $\perp$.

(1) OpenEnc(PP,C,M) $\rightarrow$ letter: This formula is for the sender to unharness secret writing proof PE for $(\mathrm{M}, \mathrm{C})$.OpenDec(PP, SK,C,M) $\rightarrow$ atomic number 46: This formula is for the receiver to unharness cryptography proof $\mathrm{PD}$ for $(\mathrm{M}, \mathrm{C})$.

(1) Verify(PP,C,M, PE, PD) $\rightarrow$ : This formula is employed to verify the correctness of letter and atomic number 46

\section{Conclusion:}

In this work, we have a tendency to projected a confutative $\mathrm{CP}-\mathrm{ABE}$ theme to create Associate in Nursing audit-free cloud storage service. The deniability feature makes coercion invalid, and also the ABE property ensures secure cloud information sharing with a fine-grained access management mechanism. Our projected theme provides a attainable thanks to fight against immoral interference with the proper of privacy. we have a tendency to hope additional schemes will be created to guard cloud user privacy In this work, we have a tendency to projected a confutative $\mathrm{CP}-\mathrm{ABE}$ theme to create Associate in Nursing audit-free cloud storage service. The deniability feature makes coercion invalid, and also the $\mathrm{ABE}$ property ensures secure cloud information sharing with a fine-grained access management mechanism. Our projected theme provides a attainable thanks to fight against immoral interference with the proper of privacy. we have a tendency to hope additional schemes will be created to guard cloud user privacy 


\section{References}

[1] Po-Wen Chi and Chin-Laung Lei, Member, IEEE, "Audit-Free Cloud Storage via Deniable Attribute-based Encryption", IEEE Transactions on Cloud Computing, 2015.

[2] V. Goyal, O. Pandey, A. Sahai, and B. Waters, "Attribute-based encryption for fine-grained access control of encrypted data," in ACM Conference on Computer and Communications Security, 2006, pp. 89-98.

[3] J. Bethencourt, A. Sahai, and B. Waters, "Ciphertext-policy attribute-based encryption," in IEEE Symposium on Security and Privacy, 2007, pp. $321-334$.

[4] B. Waters, "Ciphertext-policy attribute-based encryption: An expressive, efficient, and provably secure realization," in Public Key Cryptography, 2011, pp. 53-70. 